\title{
Prosthodontic Management of a Completely Edentulous Patient with Microstomia and Flat Ridge Mandible: A Case Report
}

\author{
Michael Josef Kridanto Kamadjaja and Fatty Nada Pertiwi \\ Department of Prosthodontic, Faculty of Dental Medicine, Universitas Airlangga, Surabaya, Indonesia
}

\begin{abstract}
It is difficult to make impression and fabricate conventional dentures for patients with microstomia and flat ridge. This is a case report of 53-year-old female with limited mouth opening $(25 \mathrm{~mm})$ who had been wearing denture for 7 years and which was recently broken. Close mouth with suction impression method was used. Proper diagnosis and appropriate set of procedures and methods were needed for this patient. The conventional complete denture fitted with closed mouth method for this condition resulted in retentive and stable denture which was inserted and removed easily.
\end{abstract}

Key Words: microstomia, closed mouth method, flat ridge, complete denture

\section{INTRODUCTION}

In prosthodontics practice, limited mouth opening in patients is a common occurrence. Prosthetic rehabilitation of patient with limited mouth opening due to any causal factors, causes difficulties at all stages of management, from the preliminary impression to the insertion of prosthesis. ${ }^{1}$

Microstomia is defined as an abnormally small mouth opening that, if permanent, can affect diagnosis or dental treatment. Adequate or normal mouth opening is essential for facial expression, oral feeding and speech. In addition, microstomia can cause difficulty in maintaining oral hygiene. ${ }^{3}$

Treating microstomia is complicated and depends on the causes that produce it. Various surgical procedures have been done mostly to change the soft tissue, although it is possible to use several non-surgical procedures such as oral physiotherapy exercises. ${ }^{3}$

Microstomia can be due to various etiologies, one of which is scleroderma. Scleroderma is a connective tissue disorder of the skin, joints, and sometimes internal organs. The facial skin and oral mucosa become thin and tight, and wrinkles disappear, resulting in a mask-like appearance and reduced mouth opening. ${ }^{3}$

Limited mouth opening was found in $80 \%$ of patients

Paper presented at the Joint Scientific Meeting in Special Care Dentistry, July 5, 2019, Amerta Room, 4th Floor, main campus of Universitas Airlangga, Surabaya, Indonesia.

Corresponding author: Michael Josef Kridanto Kamadjaja Department of Prosthodontic

Faculty of Dental Medicine

Universitas Airlangga

Jl. Mayjen. Prof. Dr. Moestopo No. 47 Surabaya 60132 - Indonesia

Email: michael-j-k-k@fkg.unair.ac.id observed by Marmary et al. Hand deformities (Raynaud's phenomenon), together with loss of tactile sensation, make insertion and removal of dentures difficult. ${ }^{3}$

Patients with microstomia who need to wear removable dental prosthesis often face difficulty of being unable to insert or remove the prosthesis because of the restricted opening of the oral cavity. In such patients, it is difficult to 
make impressions and fabricate dentures using conventional method. During impression procedures, wide mouth opening is required for proper tray insertion and alignment that is not possible in patients with limited mouth opening. Making an impression presents the initial difficulty in prosthetic rehabilitation in microstomia patients. ${ }^{4}$

Total edentulous is a major health problem that affects quality of life. The success of using complete denture depends on many variables, but three factors stand out in terms of functional success: support, stability, and retention. To obtain this, the final impression is very important in terms of recording the entire functional denture bearing area. Some morphological factors can increase the difficulty of making satisfying complete denture. Thus, the aims of prosthodontics treatment include: providing lip support, improving articulation and function, and regaining good aesthetics. ${ }^{4}$

A patient with flat ridge needs complete denture for chewing, speaking and improving appearance. A severe atrophy of the alveolar ridge, both in the maxilla and mandibula, represents a great challenge. This atrophy, which occurs after tooth extraction, is more rapid when the patient wears denture installed for a long time. Due to the massive resorption of this ridge, an excessive movable soft tissue, in the form of a flabby ridge may appear; its presence results in difficulty in getting an accurate, good quality, and suitable denture. $^{2}$

Accurate impression is needed to make complete denture if it follows a flat alveolar ridge. The principle of impression using compression is to achieve well basis mucosal on ridge, with light compression on the upper most ridge and to cover submucosal tissue. There are two methods of impression with compression involving open and closed mouth. Close mouth method could develop physiological strength of muscle trimming during the border molding; the impression could record the compressed soft tissue to achieve good result. In this technique, patient cooperation is really needed. ${ }^{2}$

The aim of this article is to manage a completely edentulous patient with flat ridge and microstomia due to scleroderma using a conventional complete denture with closed mouth impression technique.

\section{CASE REPORT}

A 59-year-old female patient with microstomia due to scleroderma reported to the Department of Prosthodontics of RSGMP UNAIR with the purpose of having new dentures. The patient had been using complete denture (maxilla and mandible) since 7 years ago but the denture had been broken and was not comfortable to wear.

On extraoral examination, the patient had completely edentulous flat ridge traced to a history of scleroderma since 15 years ago. She had limited mouth opening of about $25 \mathrm{~mm}$ (Figure 1). There were no TMJ abnormalities. The patient presented with typical clinical features of scleroderma such

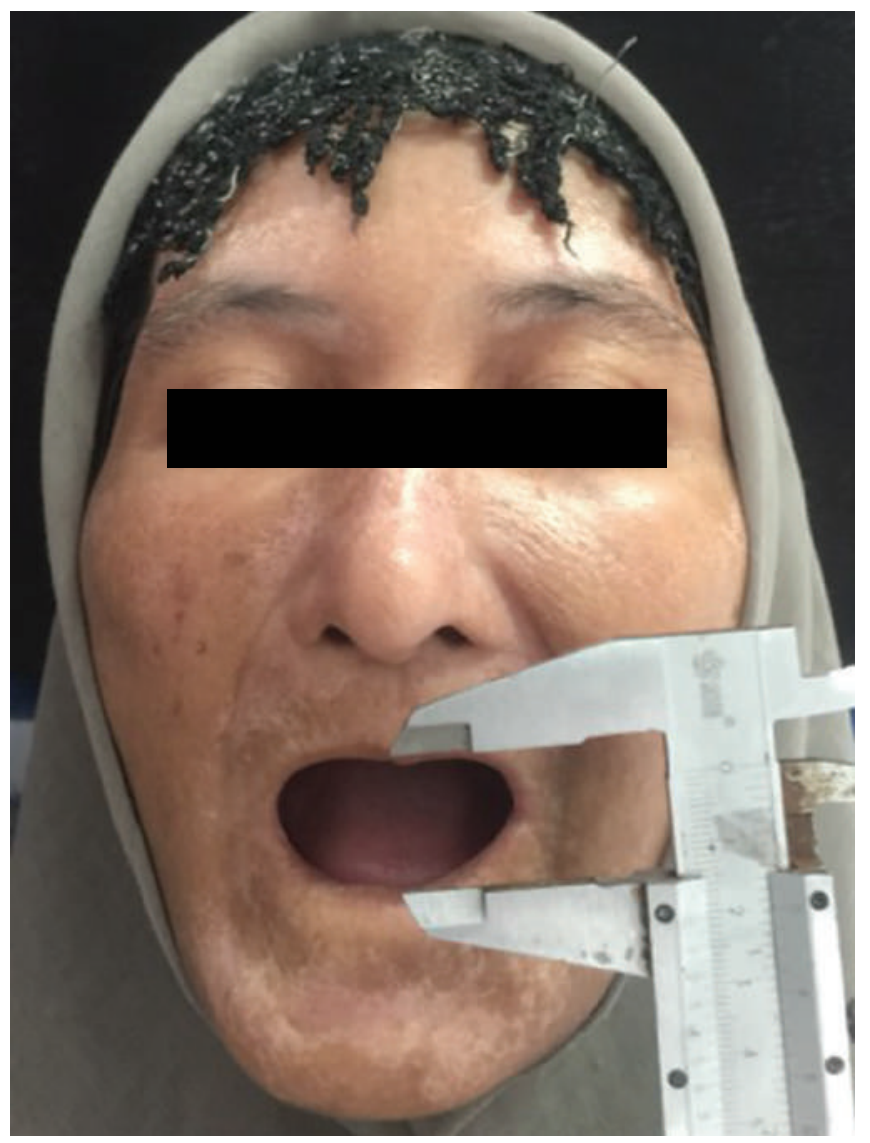

Figure 1. Mouth opening of the patient.

as claw-like hand and atrophy of fingers tips along with loss of tactile sensation. This posed a practical challenge for the fabrication of the complete dentures in a conventional way.

Intraoral examination showed abnormal ridge height and width, with flat ridge in the lower jaw. There were remaining roots of 33 and 34 with 3 degrees mobility. The upper jaw ridge was ovoid. There was no torus but exostosis was seen. On palpation, the mucous membrane was firm. There was minimal to zero resiliency of the tissues. (Figure 2).

On radiographic examination; there was a diffused radiolucency below 33 and 34 roots. Root canals of 33 and 34 were radiopaque. The decreased alveolar bone in the lower jaw indicated bone abnormality called flat ridge (Figure 3). Based on the above condition, clinical diagnosis was established; missing teeth were $18,17,16,15,14,13,12,11,21,22,23$, $24,25,26,27,28,38,37,36,35,32,31,41,42,43,44,45,46$, 47,48 , with chronic apical periodontitis and gangrene in the radices of 33 and 34, and microstomia due to scleroderma.

In this case, complete denture with impression using the closed mouth technique was recommended because the patient had resorption of lower flat jaw. The initial treatment was performed with the extraction of teeth 33 and 34 .

Preliminary passive impression for maxillary arch was taken using the patient's old denture because the smallest size of stock tray could not be inserted; mandibular primary 

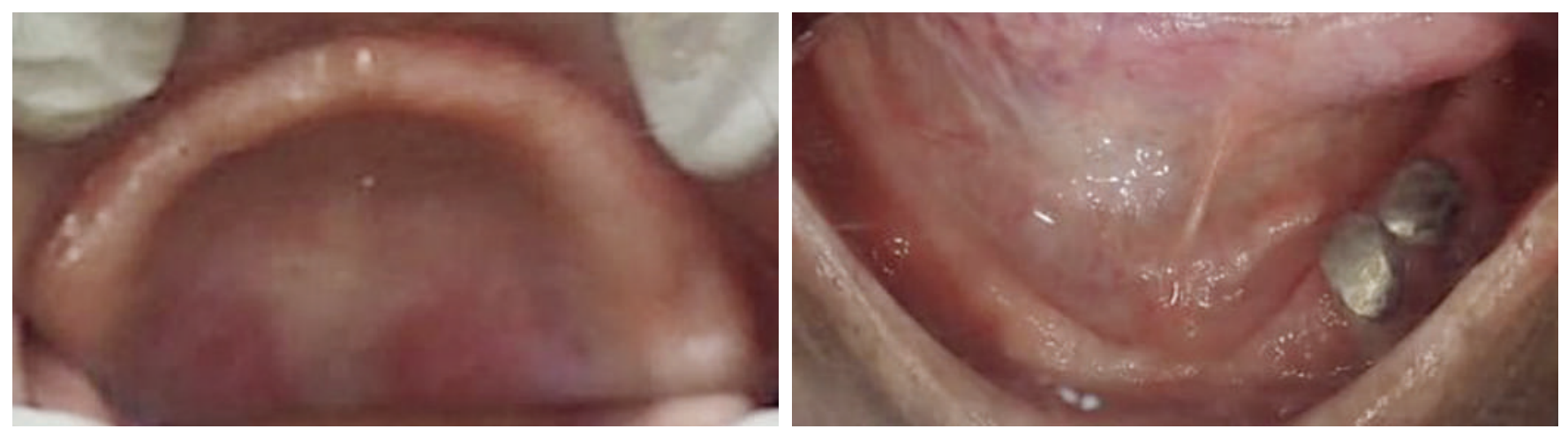

Figure 2. Pre-operative patient's intraoral examination.

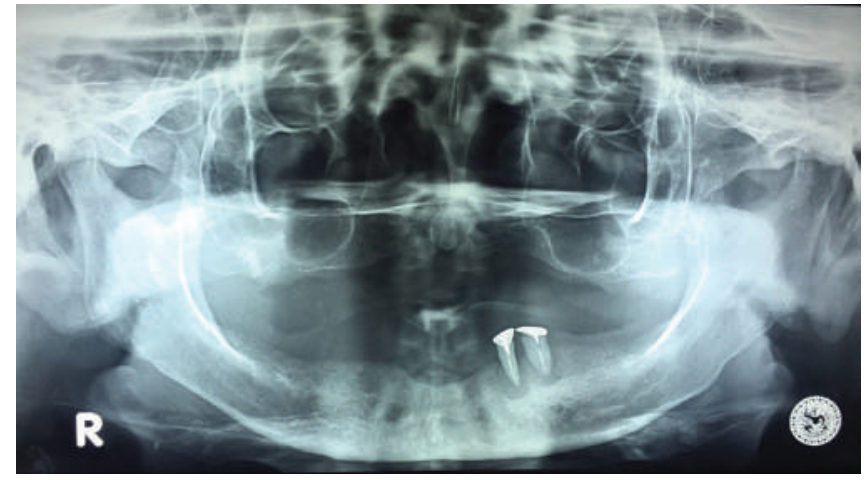

Figure 3. Pre-operative radiograph.

impression was taken using the smallest size stock tray which was inserted properly. The impression was made with alginate impression material and passive impression was used for better results; after the impression disinfection, the primary cast was obtained.

A polymerizing acrylic resin custom tray was fabricated for the maxillary and mandibular arch. Border molding and final impression was made with polyvinyl siloxane added to silicon impression material. The impression (polyvinyl siloxane) was filled up with type IV dental stone to form the working model.

After obtaining the maxillary and mandibular master cast, from the master cast acrylic base, the bite wax was made. The height and bite position was sought; this was not fixated because of the limited mouth opening. The next step involved the suction method. Four pillars were made on the lower jaw's bite wax for the closed mouth functional impression using the suction method; part of the wax of the lower jaw was cut off. The pillars were used to facilitate the patient's sucking while doing the impression. The suction method was used for impression on the the flat ridge. The function of the suction method, in patients with flat ridge condition, was to achieve sealing of the entire borders, a simple technique for enhancing the retention and stability of denture (Figure 4).

The mucocompressive impression with elastomer material was then obtained. Patient was instructed to move her lips as if she was sucking and saying the letters "O" and "E"; then the tongue was moved to the right and left. Elastomer impression was placed on the upper bite wax and then returning to the mouth, followed by the lower bite wax and were fixated together. The impression process involved closed mouth and suction method using compressive material (Figure 5). After making the final impression using the suction method, the bite registration followed. The process was continued by filling with hard gypsum; the working model was now formed and put on an articulator according to the guidelines of the close mouth impression result.

Patient with limited mouth opening poses a very difficult and challenging task to taking impression. The operator should know the good position of the mold so it can be inserted properly. The impression will not be as good as in a patient with normal mouth opening, because of the limited opening of the mouth of about $25 \mathrm{~mm}$. The impression touching parts of the mouth that should not be, finally the part that should be recorded is not perfectly recorded. The following step, teeth arrangement was done and adjusted on the patient (Figure 6).

Over bite and over jet of the anterior teeth should be adjusted. Close attention should be given to the curve of spee as seen from sagittal side and the curve of monsoon of the transverse side of the posterior tooth arrangement.

If the patient was satisfied with the denture wax, the next step involved contouring, acrylic processing and polishing. The next step would be adjusting the acrylic denture on the patient. Occlusal record was performed for occlusal correction, then followed by selective grinding, polishing and finally insertion of the denture to the patient (Figure 7).

The instructions given to the patient after the denture insertion include: for the first 24 hours only drinking and speaking would be allowed; eating would not be allowed. The denture was recommended to be used at night with follow-up on the next day (Figure 8).

On the first day of follow up, the patient did not have any complaint. The instruction for the next three days was to use the denture for eating something soft, drinking, and speaking. The denture should be removed at night and soaked in water, with the purpose that the mouth tissue could rest. 

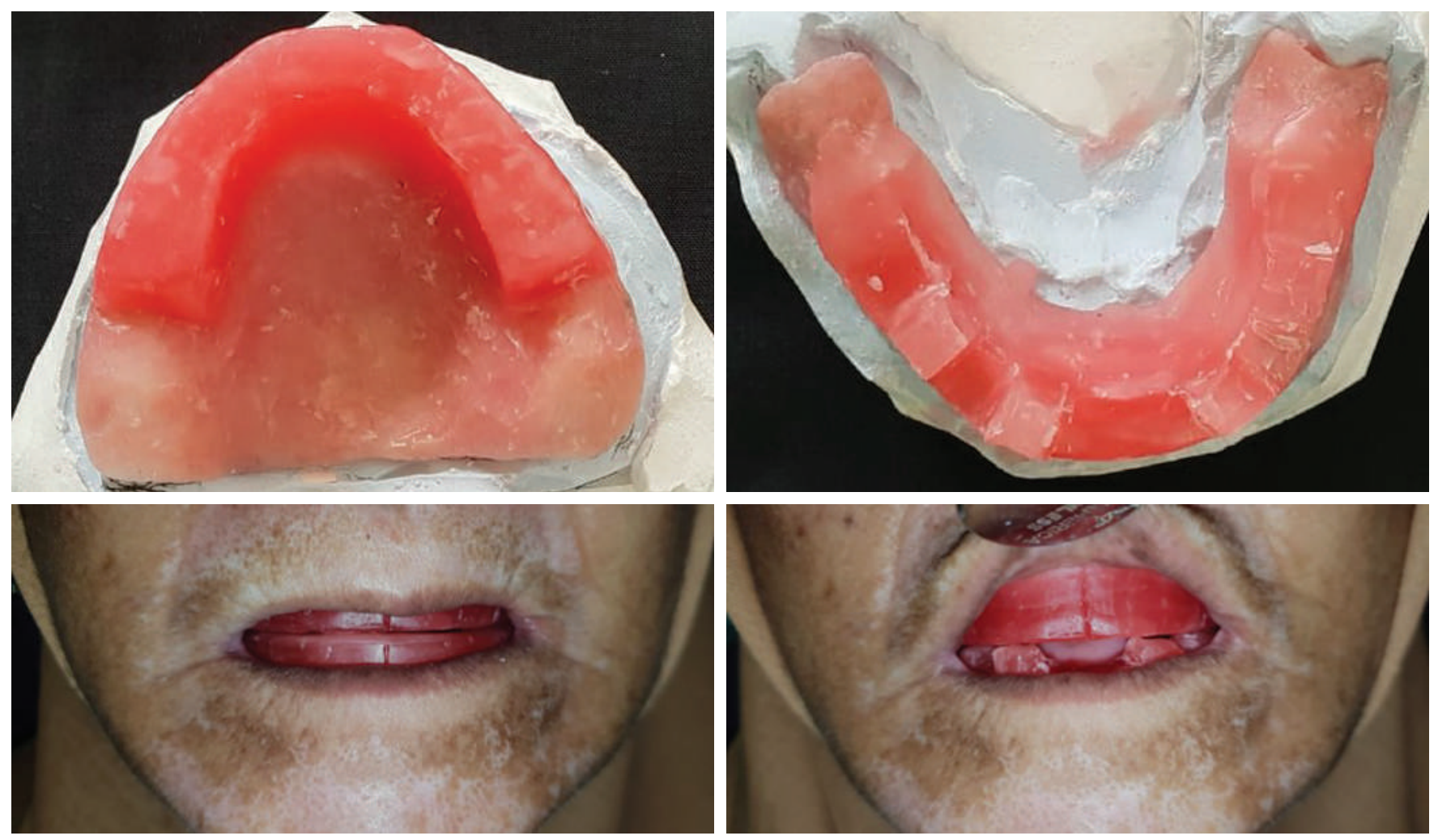

Figure 4. Bite wax with four pillars on lower jaw.
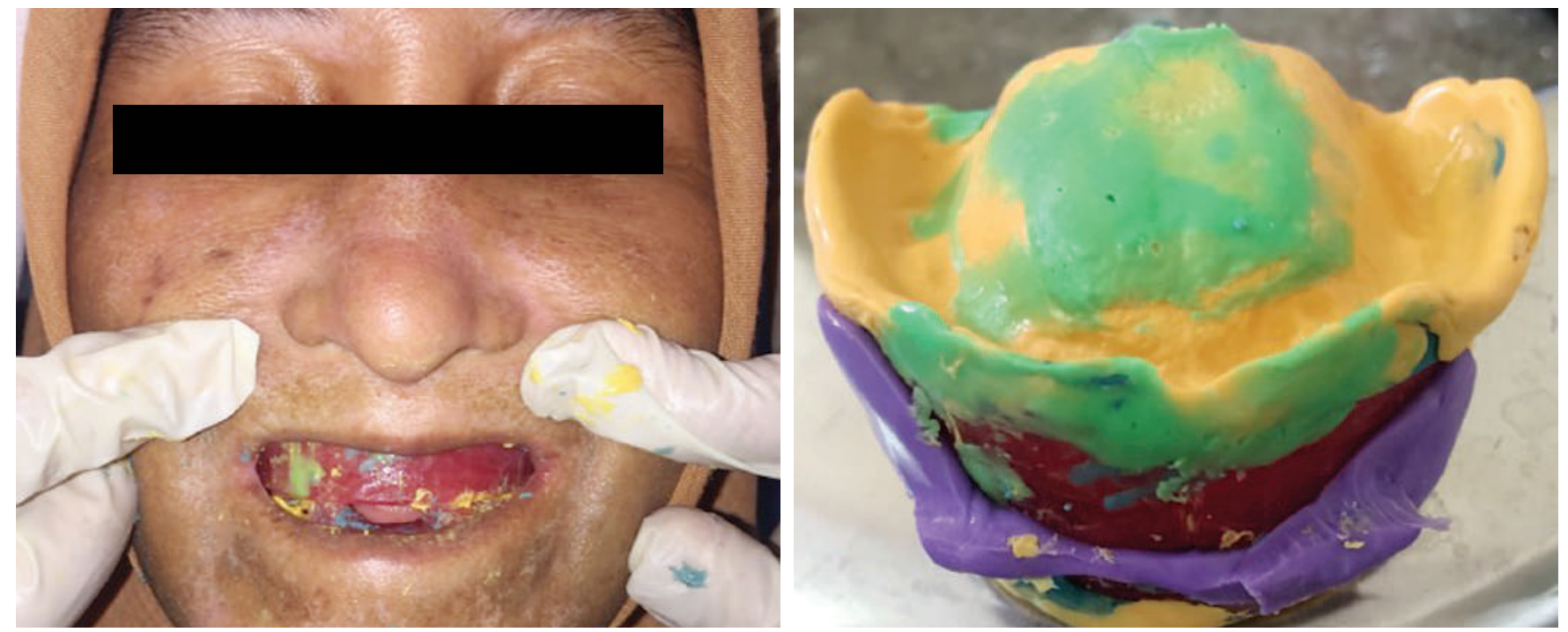

Figure 5. The impression result of close mouth technique.

On the second follow up, the condition of upper and lower jaw denture was stable and retentive, but the patient complained of pain in the posterior region of lower jaw when she was eating. The operator used articulating paper to check the occlusion, showing bold spot on the posterior teeth. Then the posterior teeth region of lower denture was ground and polished. The instruction given was still similar to the first control visit.

On the third follow up, the patient still complained of pain in the posterior region of the lower jaw, but the complete denture was retentive and stable so further grinding and polishing was done. The instruction was still similar to the second control visit if there was any complaint; the patient was advised to have regular control visit every 6 months. 

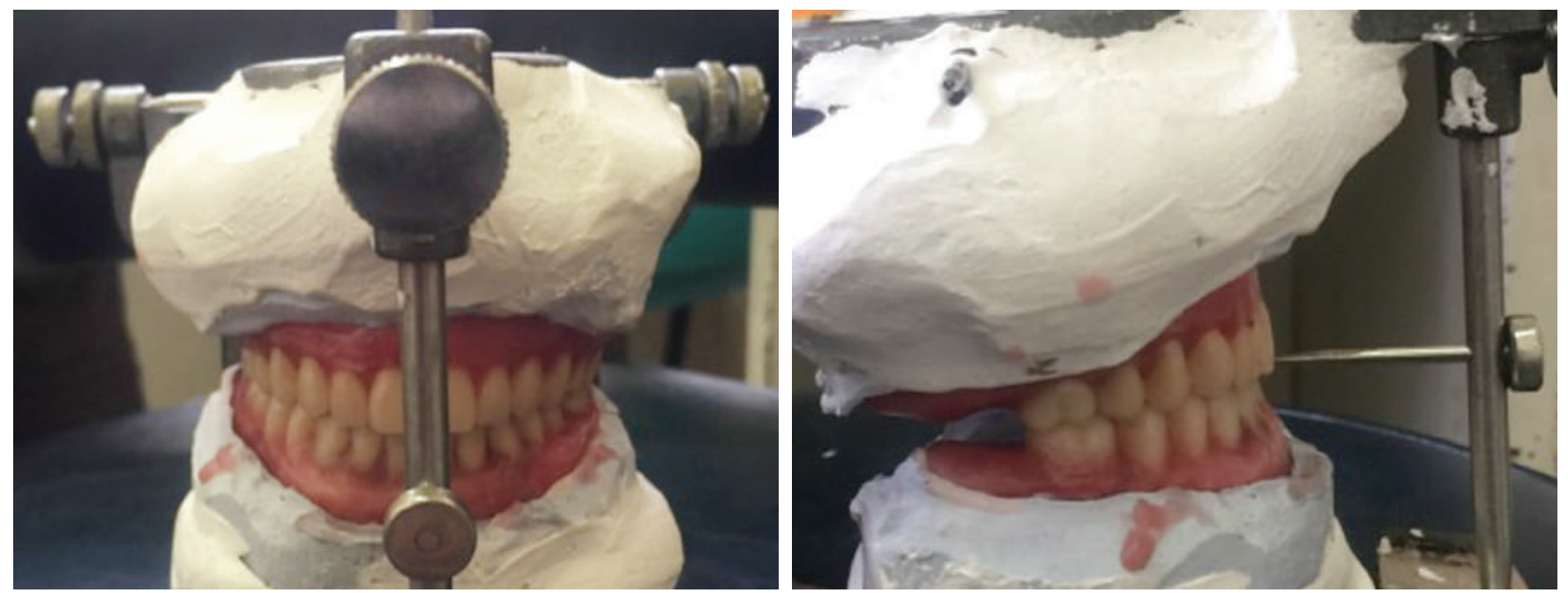

Figure 6. The teeth arrangement on articulator.
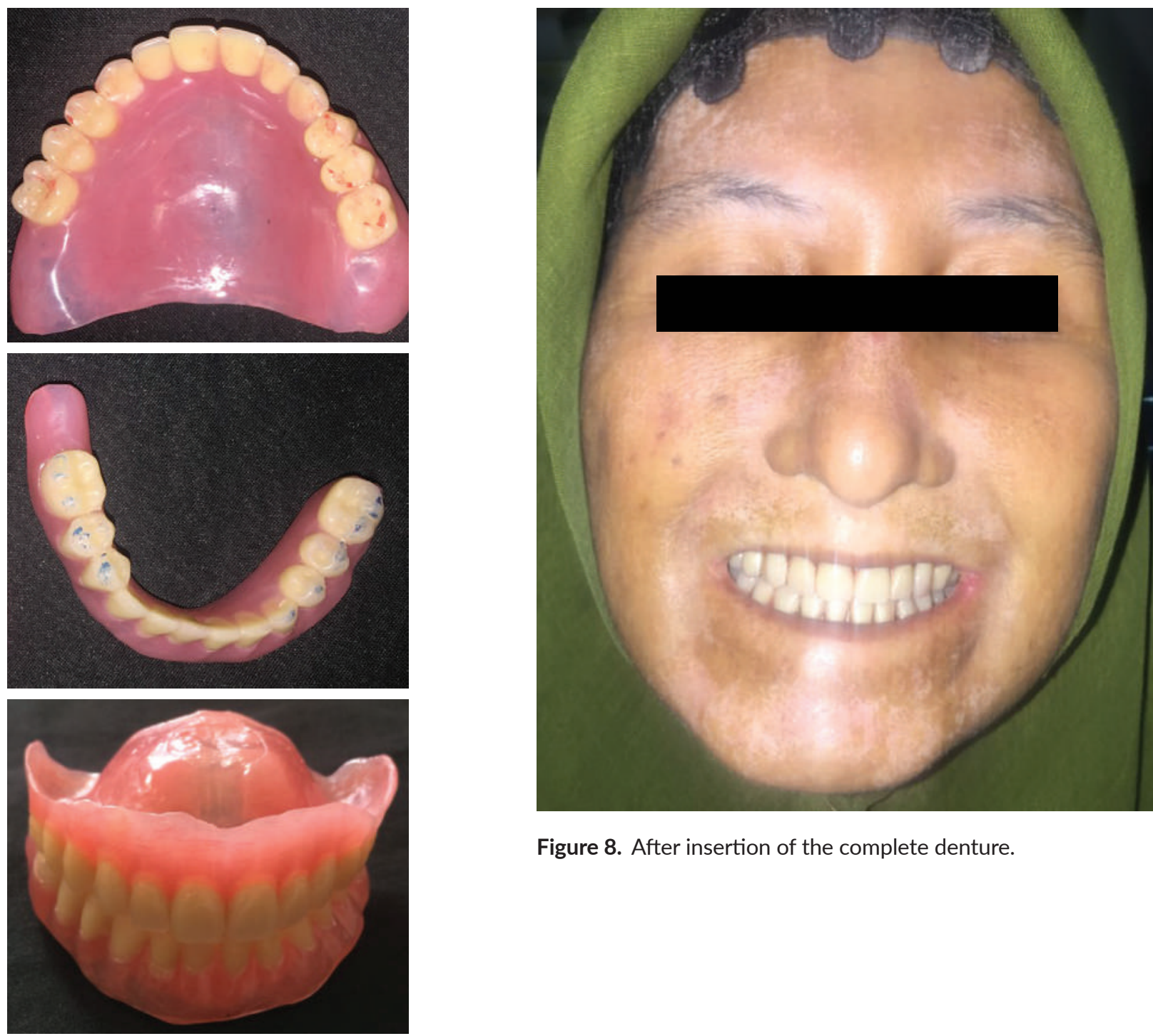

Figure 8. After insertion of the complete denture.

Figure 7. Complete denture. 


\section{DISCUSSION}

Microstomia is defined as an abnormally small mouth opening that affects the diagnosis and dental treatment. Microstomia is a condition that may be caused by multiple and varied situations; clinical diagnosis of this condition has not yet been clearly defined by the right-size criteria. ${ }^{3}$

Limited mouth opening can be an obstacle to maintaining oral hygiene and proper tooth appearance. Dental problems are common in patients with microstomia. ${ }^{3}$

Dental management of patients with microstomia is a challenge for dentist to make a correct diagnosis in several dental pathologies due to limited mouth opening. However, the problem of getting the right diagnosis can be solved using panoramic radiographs. ${ }^{1}$

Microstomia is common in patients suffering from scleroderma. This condition causes problem during each step of prosthetic reconstruction starting from the selection of the primary impression tray to insertion of the dentures. Several methods of constructing sectional special trays have been discussed in literature. Various techniques to make preliminary impression for patient with limited mouth opening have been included: sectional stock tray system described by Robert J Luebke, flexible tray made with silicone putty, and flexible tray used for fluoride application. In such condition, a semi-rigid silicone putty impression material is generally used as impression tray for preliminary impression. But in this case, preliminary impression was made with the patient's old denture for upper jaw and the smallest size stock tray for lower jaw. ${ }^{1}$

There are three factors for making a good complete denture, namely, support, stability, and retention. To obtain this, the final impression is very important in terms of recording the entire functional denture bearing area. In this case, to make a good complete denture of that flat ridge case on lower jaw, it was needed to apply impression using closed mouth technique and suction method. ${ }^{2}$

Patient with flat ridge needs complete denture for chewing, speaking, and improved appearance. Due to the massive resorption of this ridge, an excessive movable soft tissue, flabby ridge may appear; its presence results in difficulty in getting an accurate, good quality and suitable denture. To increase retention, the impression with mucocompressive material is required to be able to compress the mucosa so it could produce an accurate impression. ${ }^{2}$

The impression using closed mouth method is usually used for flat ridge patients, especially the mandibular flat ridge. Closed mouth with mandibular suction method is the most important aspect in the process because it can seal entire borders to obtain retention and stability. In this case, after insertion of the complete denture, patient was satisfied with the denture. The patient felt her denture was retentive, stable, and comfortable. Patient could chew food, speak normally and she has better performance, even if she must continue to adapt to her new denture. ${ }^{2}$

It can be concluded that to make a complete denture in the management of a flat ridge case on the upper and lower jaws, it is necessary to apply the impression using a closed mouth technique and mucocompressive impression to obtain retention and stability. ${ }^{4}$

\section{CONCLUSION}

Even though it is difficult to achieve all the aspects of prosthodontics treatment in a patient with microstomia and flat ridge mandible, with improved working skills and techniques we can still accomplish our goal. Closed mouth with suction impression method can be used for the conventional complete denture of this patient. Other prosthodontics treatment like implants, obturators, and cast partial dentures can be done for this type of patients with slight modifications in all the procedures. Still, dental treatment in patients with microstomia is difficult. The preventive approach would be most suitable dental treatment for this patient and with careful treatment planning and designing, many of the apparent clinical difficulties can be overcome. All the procedures were intended for better function, health, aesthetics, and overall patient's welfare.

\section{Statement of Authorship}

All authors participated in data collection and analysis, and approved the final version submitted.

\section{Author Disclosure}

All authors declared no conflict of interest.

\section{Funding Source}

None.

\section{REFERENCES}

1. Chairamana Sandeep O, Swetha Hima Bindu B, Sreedevi K, Sai Prasad. Prosthodontic management of a completely edentulous microstomia patient. J Orofac Sci. 2014; 6(1):65-8.

2. Abe J, Kokubo K, Sato K. Mandibular Suction Effective Denture and BPS: A Complete Guide. Quintessence Publishing; 2012.

3. Silvestre-Rangil J, Martinez-Herrera M, Silvestre FJ. Dental management of patients with microstomia. A review of the literature and update on the treatment. J Oral Res. 2015; 4(5):340-50.

4. Satpathy A, Gujjari AK. Complete denture in a microstomia patient. J Clin Diagnostic Res. 2015;9(5):16-8. 